\title{
Gleicher Lohn für gleiche Leistung
}

Alice Salomon

Follow this and additional works at: https://scholarsarchive.byu.edu/sophnf_essay

Part of the German Literature Commons

\section{BYU ScholarsArchive Citation}

Salomon, Alice, "Gleicher Lohn für gleiche Leistung" (1906). Essays. 674.

https://scholarsarchive.byu.edu/sophnf_essay/674

This Article is brought to you for free and open access by the Nonfiction at BYU ScholarsArchive. It has been accepted for inclusion in Essays by an authorized administrator of BYU ScholarsArchive. For more information, please contact scholarsarchive@byu.edu, ellen_amatangelo@byu.edu. 


\section{Gleicher Lohn für gleiche Leistung (Essay, Scientific Work, 1906)}

Vor mehr als zwei Menschenaltern hat Thomas Hood das Los einer Näherin mit den Worten geschildert:

"Schaffen - Schaffen - Schaffen -

Und der Lohn! Ein Wasserhumpen,

Eine Kruste Brot, ein Bett von Stroh,

Dort das morsche Dach - und Lumpen!

Ein alter Tisch, ein zerbrochener Stuhl,

Sonst nichts auf Gottes Welt!

Eine Wand so bar - 's ist ein Trost sogar,

Wenn mein Schatten nur drauf fällt."

Diese Worte können noch heute als Gleichnis für die Entlohnung der meisten Frauen gelten.

Welchem Beruf sich die Frauen auch heute zuwenden mögen, überall wird es ihnen schwer gemacht, sich durch ihre Arbeit ausreichende Mittel zum Lebensunterhalt, zu einer anständigen Lebensführung zu verschaffen. Der alte volkswirtschaftliche Lehrsatz, daß die Arbeit die Quelle allen Reichtums ist, hat für die Frauen schlechthin keine Geltung. Denn die Frauen, die ihr Leben lang gearbeitet haben, sind meist nicht reich geworden, und die Frauen, die reich geworden sind, haben das meist nicht durch ihre Hände oder ihres Kopfes Arbeit erreicht. Die Löhne der Frauen in den verschiedensten Berufssphären zeigen die traurige Gemeinsamkeit eines auffallenden Tiefstandes.

Diese Tatsache hat dazu geführt, daß man sich in allen Kreisen die Frage nach den Ursachen der niedrigen Frauenlöhne vorlegt. Männer haben die Frauenarbeit bekämpft, weil sie durch die Frauen unterboten wurden, weil sie in ihnen unlautere Konkurrentinnen, Lohndrückerinnen sahen. Die Frauen aber haben sich bitter über die Ungerechtigkeit der ihnen gezahlten Löhne beklagt. Es ist sogar die Behauptung aufgestellt worden, daß man den Frauen nur geringere, schlechtere Löhne als den Männern zu bieten wagt, weil sie politisch rechtlos seien. Aus solchen Betrachtungen ist die Forderung "gleicher Lohn für gleiche Leistung", die sich im Programm der verschiedensten Frauenvereine und politischer Parteien findet, hervorgegangen.

Schon aus der Formulierung dieser Forderung geht hervor, daß Frauen und Männer für gleiche Leistungen nicht immer gleich bezahlt werden, und neben der Konstatierung dieser Tatsache schließt der Ausspruch eine Forderung ein, die aus dem Prinzip der Gerechtigkeit hervorgeht. Aber der Gedanke, von dem man sich bei dem Aufstellen dieser Forderung leiten läßt, der Gedanke der Gerechtigkeit, steht zwar im engsten Zusammenhang mit der leitenden Idee der Frauenbewegung, die für die Frauen

Gleichberechtigung in der Ausbildung und Ausübung aller Berufe, Gleichberechtigung vor dem Gesetz und dem Staat verlangt und erhofft, alle diese Forderungen durchzusetzen, wenn nur der Glaube an ihre Gerechtigkeit verbreitet werden kann. Aber in Bezug auf das Postulat eines gleichen Lohnes kann dieser Gedanke nicht zutreffend sein. Dieser kann nicht - wie vielleicht noch die Frauen der vorigen Generation glaubten - durch einen Druck auf die Arbeitgeber durchgesetzt werden, denn auf dem Arbeitsmarkt herrschen nicht die Ideen der Gerechtigkeit, sondern die des wirtschaftlichen Vorteils.

Der wirtschaftliche Vorteil aber bedingt, daß die Arbeitgeber die billigste Arbeit nehmen, die sie finden, daß sie billige Arbeit nehmen, wo sie sie finden. Tatsächlich bot sich und bietet sich noch heute die Frauenarbeit den Unternehmern aber fast immer billiger als gleichwertige männliche Arbeit dar. Gerade die Billigkeit der Frau hat ihr Eingang auf dem Arbeitsmarkt verschafft und sie vielfach tatsächlich zu einer 
gefährlichen Konkurrentin des Mannes gemacht. Nicht nur bei Arbeiten, die sie auf Grund ihrer besseren Geeignetheit übernehmen kann, sondern auch in den Gruben und Bergwerken, auf Bauten und an Hochöfen, eben weil die Frau den Mann unterbot, weil sie als Lohndrückerin auftrat.

Deshalb muß die Forderung gleicher Lohn für gleiche Leistung in erster Linie in unseren eigenen Reihen gelten. Wir müssen unsere Bemühungen darauf richten, daß die Frauen selbst die Ursachen beseitigen, die zu einer ungleichen Entlohnung von Mann und Frau, zu einer geringeren Lohnforderung der Frauen geführt haben.

Die Ursachen der ungleichen Entlohnung sind mannigfacher Art. Zunächst läßt sich feststellen, daß nur in ganz seltenen Fällen die Frauen tatsächlich die gleiche Arbeit wie Männer tun. Namentlich in der Fabrik, wo Mann und Frau oft nebeneinander im selben Arbeitssaal stehen, dieselben Maschinen bedienen, handelt es sich entgegen den allgemeinen Anschauungen um ganz verschiedenartige Handreichungen und Dienste, die von beiden geleistet werden. Die fortschreitende Arbeitszerlegung hat auch hier, wo keine Arbeitsteilung für die Geschlechter durch Gesetz und Recht vorgeschrieben war, zu einer fortschreitenden Differenzierung geführt. Man kann wohl sagen, daß am gleichen Ort und zur gleichen Zeit nur ganz vereinzelt, nur in wenigen Ausnahmefällen die gleiche Arbeit von Mann und Frau getan wird.

Es sind nicht immer Unterschiede in der Begabung, in der körperlichen Verschiedenheit von Mann und Frau, die zu dieser Arbeitsteilung führen. Sondern vielfach nimmt die Frau eine Stellung ein, für die eine besonders geringe Lehrzeit notwendig ist. Das Vordringen der Frauen auf neue Arbeitsgebiete, das die letzten drei Jahrzehnte gebracht haben, steht in einem engen Zusammenhang zu der Möglichkeit, zahlreiche ungelernte Arbeitskräfte zu verwerten, die durch die moderne Entwicklung von Handel und Gewerbe herbeigeführt worden ist. Als die Maschine die Muskelkraft der Männer - und auch vielfach die gelernte Arbeit - entbehrlich machte, spannte die Industrie Frauen und auch Kinder - ungelernte Arbeitskräfte - in ihren Dienst. Ganz ähnlich lag es in anderen Berufsarten. Den Eintritt in das kaufmännische Gewerbe, in den Staatsdienst - als Post- und Bahnangestellte - erlangten die Frauen in einer Zeit fortschreitender Arbeitszerlegung, die die Heranziehung von wenig oder garnicht vorgebildeten Kräften ermöglichte. Auch zu Lehrerinnen an öffentlichen Schulen wurden Frauen zuerst berufen, nicht weil man ihre besondere Eignung für dieses Arbeitsfeld erkannte, sondern weil es an männlichen Bewerbern fehlte und man daher mit schlechter vorgebildeten Lehrkräften - damals waren die Frauen schlechter vorgebildet - vorlieb nehmen mußte. Überall hatten die Frauen den letzten Platz einzunehmen, die niedrigste und schlechtest bezahlte Arbeit zu tun, die besseren Plätze waren überall von Männern besetzt. Unter diesem Zeichen drangen die Frauen in die Erwerbsarbeit ein.

Aber auch wenn man von den Unterschieden in den Leistungen absieht, ist die Bezahlung der Frauen eine schlechtere als die der Männer. Man kann sogar feststellen, daß da, wo eine Frau tatsächlich ausnahmsweise die gleiche Arbeit wie ein Mann tut, ihr meist nicht der gleiche Lohn gezahlt wird. Wo aber Unterschiede in der Quantität oder Qualität der Leistung vorhanden sind, da eigentlich setzt erst die ungleiche, ungerechte Entlohnung ein. Hier wird die Frau nicht entsprechend der geringeren Leistung, sondern nach einem besonderen Maßstab bezahlt.

Aber auch diese Erscheinung ist erklärlich, wenn man die kurze Vergangenheit der Frauen auf dem Arbeitsmarkt verfolgt; wenn man nachforscht, warum sie ihre Forderungen niedriger als der Mann stellen mußten. Jahrhunderte lang haben die Frauen vorwiegend im Haus für die Familie geschafft. Ein Lohnverhältnis, das in Geld ausgedrückt wurde, blieb den meisten fremd. Wohl waren sie gewohnt oft schwere und aufreibende Arbeit zu tun, aber sie taten es aus Liebe zu ihren Angehörigen oder aus Pflichtgefühl; vielleicht auch, weil sich ihnen keine andere Existenzmöglichkeit bot. Aber sie taten es niemals für Geld. Die Arbeit der Hausfrau wird bis auf den heutigen Tag nicht bezahlt und deshalb auch meist gering bewertet. In ihren persönlichen Bedürfnissen ist die Frau, die ein großes Hauswesen leitet, 
von ihrem Mann meist durchaus abhängig; abhängiger als die Lohnarbeiterin mit dem geringsten Einkommen. Sogar der Sprachgebrauch hat diese Frauen gewöhnt, den Mann als "Ernährer" zu betrachten, auch wo gar keine Veranlassung dazu vorliegt, wo sie selbst wichtige und unentbehrliche Arbeit tun. Es ist demnach kein Wunder, daß die Frauen, als die bittre Not einer schweren Zeit sie zur Erwerbsarbeit drängte, ihre Arbeitskraft niedrig bewerteten, daß sie sie um jeden Preis herzugeben bereit waren. Die unbezahlte und unbewertete Arbeit der Hausfrau, die geringe Einschätzung ihrer Arbeitskraft haben die Frauen zu niedrigen Lohnforderungen veranlaßt.

Dazu kommt, daß die Frauen nicht im halben Maße wie die Männer nach einer steigenden Entlohnung streben. Das Gros der Frauen rechnet gar nicht damit, den vollen Unterhalt verdienen zu müssen. Während der Mann sich nicht damit begnügt und begnügen kann, den vollen Bedarf für sich selbst durch seine Arbeit zu erlangen, während er den Unterhalt für eine Familie verdienen will, setzt nur in den seltensten Fällen eine Frau den Familienbedarf bei ihren Lohnforderungen mit in Rechnung. Nicht einmal der volle Individualbedarf wird immer angestrebt, - denn unzählige Frauen wollen nur einen Zuschuß zum Familieneinkommen verdienen. Das sind die Ehefrauen, die pensionierten Witwen, die Haustöchter, die womöglich nur ihre Toilettenausgaben und die Unkosten für Theaterbesuche und andere Vergnügungen mit ihrem Verdienst bestreiten wollen. Mit diesen niedrigen Lohnforderungen schaden sich aber nicht nur die Frauen, die nicht aufs Verdienen angewiesen sind, selbst; sondern sie drücken die Löhne ihrer Konkurrentinnen, die von der Arbeit leben müssen, mit herunter. Denn die Lohnbildung vollzieht sich stets innerhalb der Gruppe von konkurrierenden Arbeitern; und die Anlagen, die Ausbildung, das Alter und die Lohnforderungen aller, die für einen Platz überhaupt in Betracht kommen, geben den Ausschlag für die Bedingungen und den Lohn, der schließlich zustande kommt.

Die Tatsache, daß viele Frauen und Mädchen nicht einmal den vollen Individualbedarf anstreben, daß sicherlich die Meisten nicht den Bedarf für eine Familie zu verdienen brauchen, trägt auch wieder dazu bei, daß die Frauen vorwiegend wenig qualifizierte Arbeit tun, daß sie nicht viel Zeit für die Erlernung eines Berufes opfern. Das wird noch verstärkt durch die Erfahrungstatsache, daß ein großer Teil der arbeitenden Frauen jung aus dem Beruf ausscheidet (das Durchschnittsalter der Berliner Handlungsgehilfin beträgt 21 Jahre). Die Mädchen sehen ihre Berufsarbeit häufig als Provisorium, als Übergangsstadium an, und deshalb herrscht in allen Klassen eine intensive Abneigung - der Eltern wie der Mädchen - gegen eine längere Lehrzeit. Man scheut sich für die Lehrjahre einer Tochter Geld zu opfern, dessen Zinsertrag nicht gewiß erscheint, während das Geld, das man für die Ausbildung des Sohnes verwendet, als vielversprechende Kapitalsanlage angesehen wird. Das Mädchen soll überall so schnell wie möglich einen Verdienst finden, nicht einen Beruf ergreifen; und das drückt der ganzen Frauenarbeit den Stempel des Dilettantischen, Provisorischen, Zufälligen auf und drückt die Löhne auf ein niedriges Niveau herab.

So verschlingen sich die Ursachen der ungleichen Entlohnung zu einem unentwirrbar scheinenden Knoten. Die vorwiegend ungelernte Frauenarbeit, die niedrigste Löhne zur Folge hat, hängt eines Teils zusammen mit dem unentwickelten Stadium der Frauenarbeit, mit der Notwendigkeit, auf dem Arbeitsmarkt da vorzudringen, wo am leichtesten ein Platz zu erobern ist. Sie hängt aber auch zusammen mit der Auffassung der Erwerbsarbeit, die noch in weiten Frauenkreisen den nötigen Ernst vermissen läßt. Durch die ungenügende Ausbildung leidet aber wiederum die Organisationsmöglichkeit und die Widerstandsfähigkeit der Frau. Das ist eine weitere Ursache der niedrigen Löhne. Schließlich hängt die geringe Bewertung der weiblichen Arbeitskraft - wo durch die Frauen selbst - mit der kurzen Vergangenheit der Frauenerwerbsarbeit zusammen. Nur wo es den Frauen gelungen ist für einen ganzen Stand diese Ursachen der schlechten Bezahlung zu beseitigen, die in letzter Linie in dem unentwickelten Stadium der Frauenberufsarbeit, in der dilettantischen Auffassung des Berufslebens und der ungenügenden Ausbildung der Frauen zu suchen sind, da haben sie auch ihre Gehälter und ihre Löhne 
den männlichen anzunähern vermocht. In erster Linie kann als Beispiel hierfür der deutsche Lehrerstand genannt werden, der seine vorzügliche Berufsorganisation nutzbar machte, um die Anforderungen an die Mitglieder des Standes immer höher zu stellen. Wenn beispielsweise das Wissen und Können der Volksschullehrerinnen hinter dem ihrer männlichen Kollegen nicht zurückbleibt, so ist es darauf zurückzuführen, daß durch die Initiative der Lehrerinnen die Prüfungsanforderungen für die Frauen immer höhere wurden. Auf Grund dieser Leistungen haben die Frauen dann Gehaltsaufbesserungen durchgesetzt, wie das letzte Preußische Lehrerbesoldungsgesetz sie den Frauen gebracht hat.

Nur wenn in allen Berufszweigen die Frauen in ähnlicher Weise die Anforderungen an sich selbst höher stellen; wenn sie, ebenso wie die Männer, für den Beruf erzogen und tüchtig gemacht werden - gleichviel, ob sie ihn dauernd in vollem Umfange ausüben oder nicht - dann nur können die Frauen zu höheren Stufen der Leistungsfähigkeit und zu einer gerechten Entlohnung gelangen. Es muß in den Frauen die Liebe zur Arbeit gepflegt werden, die Berufstreue und Berufshingabe, damit sie während der Dauer ihrer Berufsarbeit den ganzen Menschen einsetzen und auch den vollen Unterhalt für einen ganzen Menschen beanspruchen können. Wir müssen für den gleichen Lohn, den wir fordern, die gleiche Arbeit wie der Mann geben, und zwar nicht nur dasselbe Können, dieselbe Geschicklichkeit, sondern auch dieselbe Ausdauer, Regelmäßigkeit, Hingabe und Berufstreue. Dann wird die Frauenarbeit vordringen, wo sie geeigneter als Männerarbeit ist, nicht weil sie billiger ist. Und auch für die Frauen wird dann die Arbeit zur Quelle allen Reichtums werden.

Das Blaubuch, 1. Jg., Nr. 30, 2. Aug. 1906, S. 1167-117 